\title{
Improving practice
}

\author{
Dr lan Bentley, GP, Hill Lane Surgery, Southampton
}

\section{Summary}

Hepatitis C affects a substantial number of people in the UK. According to recent guidelines from the National Institute for Health and Clinical Excellence (NICE), up to half a million individuals are infected with the hepatitis $\mathrm{C}$ virus (HCV) in England and Wales alone. However, many HCV-infected patients will go undiagnosed, and even when they are diagnosed, effective treatment is severely restricted to only a small proportion of patients. To improve the management of hepatitis $C$ in the UK there is a clear need for greater awareness and better education both amongst the medical community and the general population. Better education will increase the proportion of patients who are diagnosed and treated and will also reduce the likelihood of a future epidemic of liver disease. Improved education will also reduce the stigma that persists in many sections of the population towards individuals with hepatitis $C$, which arises as a result of the primary route of transmission being intravenous drug misuse. Primary care has a major role to play in case-finding undiagnosed cases of hepatitis $C$ and at-risk groups can be identified from computerised records and targeted for subsequent follow up. In practice, however, it can be challenging to identify patients either in the acute or chronic phases of HCV infection, as symptoms are often subclinical or non-specific. When HCV infection is suspected or diagnosed, onward referral to a specialist clinic is required for further confirmatory diagnosis and management. Combination therapy with pegylated interferon and ribavirin is the treatment of choice for managing chronic hepatitis $C$ and can have a cure rate of up to $84 \%$ depending on the genotype of the infecting virus. Recent NICE guidance recommends pegylated interferon and ribavirin as an effective and cost-effective treatment strategy for patients with mild chronic hepatitis C. However, the treatment is associated with a multitude of side-effects and primary care has an important role to play in managing these side-effects and also in providing appropriate education for patients. More effective interdisciplinary working practices between the GP and the hospital team will allow treatment to be delivered in the most effective fashion.

\section{Introduction}

The burden of hepatitis $\mathrm{C}$ in the UK has been likened to an iceberg, with only the very tip of the infected population being known. According to NICE, there are approximately 200,000-500,000 people living with hepatitis $\mathrm{C}$ in England and Wales. However, the Department of Health in the UK has estimated that only 47,000 of these individuals received a diagnosis of hepatitis $\mathrm{C}$ in 2005. Furthermore, of the total pool of infected patients (undiagnosed and diagnosed), only a very small proportion $(\sim 1.5 \%)$ actually receive any treatment. This is due to a number of factors including doctor, patient and administrative issues.
Drugs in Context

DOI:

http://dx.doi.org// 0.7573/dic.2 I 2204

Citation: Bentley, I. Improving Practice - Hepatitis C.

Drugs in Context: e21220I. doi: $|0.7573| 37 \mid /$ dic. 212204

Copyright: this is an open access article published under the terms of the Creative Commons License Deed (CC BY-NC-ND 3.0) which allows you to share, copy, distribute and transmit the work provided it is properly attributed. You may not use this work for commercial purposes. For further information

on commercial use, contact publisher@justmedicalmedia.com or go to

www.drugsincontext.com/copyright.

NB: This article was originally published by CSF Medical

Communications Ltd (CSF) in Drugs in Context 2007;3(2):85-91.

Drugs in Context and all CSF copyrights were acquired by Just Medical Media Ltd in 2009.

\section{0}

There are approximately 200,000-500,000 people living with hepatitis $C$ in England and Wales. 


\section{Prevalence and burden of hepatitis $\mathbf{C}$ in primary care}

The worldwide prevalence of hepatitis $\mathrm{C}$ is thought to be about $1 \%$. In primary care in the UK, such a prevalence would translate into about 20 patients for each full-time GP, assuming an average GP's list size of 2,000 patients. Of these patients, the GP is likely to be aware of only a handful of cases of hepatitis $\mathrm{C}$ infection.

Hepatitis $\mathrm{C}$ is a virus about which little has been known about until the last two decades. Since then its significance in terms of its associated morbidity and mortality has been brought more sharply into focus as more information has become available and treatment options have developed and improved. Hence, there has been a push to improve knowledge both amongst the medical community and the wider population to try and reach groups at risk in order to prevent an epidemic of severe liver disease in the future.

The natural history of hepatitis C infection is such that approximately $15-20 \%$ of infected individuals will spontaneously clear the virus and, as such, no treatment will be required. Of the remaining $80 \%$ of patients, over a period of $10-20$ years about half will develop mild chronic liver disease of minimal clinical significance whilst the other half will go on to develop severe disease resulting in cirrhosis or hepatocellular carcinoma. However, it is important to note that disease progression is not linear and at present we cannot predict who will or will not progress with any great degree of certainty. Finally, there is an estimated $5 \%$ death rate directly attributable to hepatitis $\mathrm{C}$ infection.

There are a number of risk factors known to be associated with a poorer prognosis from infection with the HCV. In these groups the response to treatment may also be reduced. These risk factors include:

- increased alcohol intake

- age $>40$ years at time of infection

- coinfection with human immunodeficiency virus (HIV)

- male gender

- chronic coinfection with hepatitis B virus (HBV).

The key challenge for primary care is to identify as many cases of hepatitis $C$ infection as possible by adopting a high index of suspicion and targeting at-risk groups.
As the number of individuals infected is so substantial, there are significant human and socioeconomic costs associated with the disease. This fact should act as an important motivator in trying to eradicate this virus from as many people as possible. Clinical evidence demonstrates that, across the board, the cure rate from treatment is in the region of $50 \%$. However, with certain viral genotypes, the cure rate increases to up to $84 \%$; clearly, a viral disease that can be cured in the majority of people is somewhat of a rarity. Nevertheless, significant numbers of people will need to be reached to reduce the long-term health costs to the state, to improve quality of life and most importantly to reduce mortality. Hence, the key challenge for primary care is to identify as many cases of hepatitis $\mathrm{C}$ infection as possible by adopting a high index of suspicion and targeting at-risk groups.

The route of transmission for the virus is parenteral and patients are likely to have been infected as a result of:

- previous intravenous drug use (in $\geq 80 \%$ cases)

- receiving unscreened blood and blood products (routine screening was introduced in 1991)

- organ transplantation from an infected donor

- haemodialysis

- intranasal cocaine use

- accidental injury with needles or syringes

- fights, bites, tattooing, body piercing, acupuncture

- sharing toothbrushes and razors

- sexual contact $(\sim 1-2 \%)$

- multiple sexual partners

- transmission to the neonate (in $<7 \%$ of $\mathrm{HCV}$-infected mothers),

- occupational exposure of healthcare workers ( $\sim 2 \%$ risk following exposure).

A major challenge facing healthcare professionals is establishing an accurate diagnosis of hepatitis $\mathrm{C}$ infection. Acute hepatitis $\mathrm{C}$ is usually a subclinical illness and rarely presents as an acute hepatitic illness with overt jaundice. If hepatitis $\mathrm{C}$ is detected during the acute phase, either because of clinical illness or through screening (e.g. after a needle stick injury), then the cure rate through treatment is excellent and somewhere in the region of $90 \%$. However, the 
majority of infected patients will be detected in the chronic phase (where the virus has persisted for more than 6 months). Diagnosis can also be difficult during the chronic phase as symptoms are often non-existent or rather nebulous, resulting in a delay or absence of a diagnosis.

As more information about hepatitis $\mathrm{C}$ is placed in the public domain, we can hope that there will be a significant increase in the numbers of people being tested and diagnosed. Once hepatitis $\mathrm{C}$ is diagnosed, it is vitally important that a higher proportion of these patients are actively managed more successfully than currently is the case.

\section{Practical strategies to meet the challenge of hepatitis C}

Some patients may specifically request a screening test because they are aware they are at risk of hepatitis C. However, this is more likely to happen in settings other than the GP's surgery such as the genitourinary medicine (GUM) clinic or through drugs advisory services and other clinics. The challenge in primary care is case finding those who were or who are still at risk of infection but who have not yet taken steps to do anything about it, or those who are infected but might not consider themselves to be at risk.

Any disclosure from the patient of an obvious risk factor such as an admission of previous intravenous drug use, multiple tattoos, being a past recipient of blood products or a partner of someone infected with hepatitis $\mathrm{C}$ should automatically trigger further investigation.

More often, patients will present with wide and varied symptoms, the predominant ones being tiredness, lethargy, right upper quadrant pain and non-specific aches and pains in muscle and joints. Vague and nebulous symptoms such as these may prompt the clinician to order a number of blood tests if no other identifiable aetiology is apparent. An abnormal result in the liver profile, usually an elevated alanine transaminase (ALT), should prompt further investigation with respect to identifying risk factors for hepatitis C. It should be noted, however, that about one-quarter of all HCV positive individuals may have ALT levels within the normal laboratory range. Even if no risk factors in the patient's history are apparent, an appropriate viral screen including one for hepatitis $\mathrm{C}$ should be undertaken. Patients may like to forget the odd needle-sharing episode at university that may have taken place many years before, whilst others may be shy about their social habits for fear of discrimination. Other individuals may have simply forgotten about occasions when they may have put themselves at risk. Targeting at-risk groups via computer audit may also prove to be a useful method for increasing the numbers of individuals screened (see Clinical Audit).

Once a risk is identified, a positive RNA for HCV is required to confirm active viral infection (a positive IgG level merely indicates that a patient has been exposed to the virus and this may or may not have been cleared by the patient's own immune system). An enzyme immunoassay for hepatitis $\mathrm{C}$ can then be performed in a specialist lab. There are a number of different genotypes of hepatitis $\mathrm{C}$ (see below), and if hepatitis $\mathrm{C}$ is diagnosed the virus needs to be both genotyped and quantified as both factors have important consequences for the future management of the condition. As these tests can be costly, it is best if they are performed by the specialist clinic once the patient has been diagnosed and referred onwards.

The challenge in primary

care is case finding

those who were or

who are still at risk of

infection but who have

not yet taken steps to

do anything about it, or

those who are infected

but might not consider

themselves to be at risk.

\section{Disease management}

The decision to refer and treat is a complicated issue and is one the patient will wish to discuss with their GP. Hepatitis C is currently treated by a combination of pegylated interferon and ribavirin. Because of the toxicity associated with this treatment, it is usually undertaken in a specialist clinic most often run by specialist trained nurses working under a consultant with a special interest in hepatitis C.

Treatment follows a set protocol involving numerous attendance examinations and blood tests to monitor the disease course and the emergence of any potential side-effects. The treatment programme typically lasts for either 24 or 48 weeks depending on the genotype of the virus and whether or not there is established cirrhosis. There are several different genotypes of $\mathrm{HCV}$ and these are important because they are associated with different prognoses. Genotypes 1, 4, 5 and 6 are more difficult to eradicate and require a 48-week period of treatment. They have a cure rate defined by a sustained viral clearance (SVR) at 6 months' post-treatment of 40-50\%. In contrast, genotypes 2 and 3 are more successfully cleared with an SVR of around $80 \%$ after a shorter 24 -week treatment course. In this county, we mainly see genotypes 1-3, with genotypes 4-6 predominating in Africa and Asia. 
Once on treatment the patient is likely to be seen in general practice for either certification from work or dealing with any side-effects that have arisen.

Close liaison is important between GP and the hospital team in both directions whilst the patient remains on the treatment programme.
Treatment of hepatitis $C$ is further complicated by a multitude of potential side-effects which are often difficult for the individual. These complications may necessitate treatment in themselves or may require postponement or a reduction in treatment dose, and in a few cases, protracted absence from work.

The treatment is also costly. In the UK, funding has to be agreed with the patient's funding authority according to criteria laid down in the NICE guidelines. The current cost of medication alone for someone undergoing 48 weeks of treatment can be up to $£ 12,000$ per patient (as of 2006). However, NICE has determined that it is cost-effective to treat all patients with hepatitis $\mathrm{C}$ who fit their criteria of having significant liver damage as a result of their disease or who are sufficiently symptomatic as a result of the virus.

Consequently, there needs to be a frank and open discussion with the patient regarding all of these factors so that an informed decision can be made as to when would the best time to embark on treatment and what might be the likely course for them as an individual. In some cases, an individual's lifestyle is so chaotic that compliance with such a tightly regulated treatment programme would be virtually impossible. Alternatively, an individual may remain in a high-risk group and therefore may be at risk of reinfection once the virus is cleared. In either case, a decision to delay treatment is probably best until their personal situation changes. However, these individuals should remain under active follow up. Postponing treatment until the right parameters are in place is by far the best way to ensure full compliance with the course of treatment and hence the best chance of success in eradicating the virus. Indeed, because this approach is now common practice, compliance rates with treatment across the country are very high (in the region of $80 \%$ ), despite the difficulties a large number of patients have whilst on treatment. Once on treatment the patient is likely to be seen in general practice for either certification from work or dealing with any side-effects that have arisen.

The pegylated interferon self-administered once weekly often results in flu-like symptoms the day after administration, and the ensuing tiredness associated with this can make work difficult. This adverse effect on work can be reduced by altering the timing of the administration of this to coincide with a rest day. However, the treatment has a tendency to result in anaemia and insomnia, which may result in a deeper and more prolonged fatigue necessitating either part-time work if available or being placed on sick leave.

One of the most common side-effects from the treatment is one of mood disorder. This can take the form of insomnia, irritability, anxiety, depression and, more rarely, suicidal ideation. All patients should be screened for a previous psychiatric history before embarking on treatment and a full psychiatric evaluation may be sought if deemed necessary with appropriate support arranged if required. Antidepressants may be prescribed in those vulnerable to depression in advance of initiating treatment. However, some mood disorders arise in patients who least expect it, and they may present to their GP for help with this. Indeed, the GP with his experience of encountering all of these problems on a regular basis may be the best placed person to help with these issues.

Contraception is very important for both men and women as ribavirin is teratogenic. Secure contraception is mandatory for the length of time on treatment and for 6 months after completion of treatment. This should be established before treatment starts. Patients should be counselled that any pregnancy conceived whilst either partner is receiving treatment or in the 6-month period after treatment has been completed has the potential to result in a spontaneous miscarriage or require a termination for foetal abnormality.

Some of the other side-effects commonly seen with therapy include:

- loss of appetite and weight loss

- bowel disturbance

- dry skin, pruritis and rashes

- cough and chest infections (which may become entrenched)

- thyroid function abnormalities (which may become permanent)

- anaemia

- low platelet counts

- disturbance of calcium metabolism (hypocalcaemia)

- vasculitis.

This list is far from exhaustive and most of these problems should be detected and managed during regular visits to the specialist hospital team. However, as these visits eventually decrease to monthly intervals and as patients often live a long way from their treatment centres they may present with any of these problems to their GP. Therefore, close 
liaison is important between GP and the hospital team in both directions whilst the patient remains on the treatment programme. This will consist of a regular discharge summary for every attendance during treatment which lists any complications and treatments for complications, and a named nurse responsible for the patient so that should problems arise both the patient and the doctor knows who the first point of contact is.

Part of the treatment programme also involves educating the patient about their condition and alleviating their fears with respect to lots of questions surrounding daily interaction with others. Each patient should be given a simple booklet outlining the answers to questions that often arise. This booklet backs up additional information they are given in the clinic before treatment is initiated and whilst the treatment programme is ongoing.

There is so much for an individual to take in when they are first diagnosed let alone dealing with the emotional impact such a diagnosis conveys. There are some important things to press home when communicating with patients and some important myths to dispel. For example, it is important to emphasise that HCV is not spread by kissing, hugging, sneezing, coughing, food or water, sharing eating utensils or drinking glasses, or casual contact. Patients should not be excluded from work, school, play, childcare or other settings because of an HCV positive infection status.

Equally, there are some important messages that should be communicated to infected patients to ensure they minimise the risk of transmitting the virus to others. Patients confirmed with HCV should not:

- donate blood, organs or semen

- share toothbrushes or razors

- expose their cuts and skin lesions

- share items that might have blood on them.

Infected patients should also be educated as to the possibility of sexual transmission. Persons with a long-term partner will not need to substantially change their sexual practices though they should discuss with their partner the potential risk (low but not absent) of sexual transmission. Counselling and testing of partner should be individualised and may offer reassurance to the couple. Some couples may decide to use barrier precautions to limit the risk further. The implications of perinatal transmission of $\mathrm{HCV}$ should also be considered.

\section{Patients' expectations and perspectives}

Many patients remain undiagnosed or may present to places other than their family doctor, possibly as a result of the stigma they perceive is associated with the disease. The stigma surrounding hepatitis $\mathrm{C}$, which still exists in the general population and amongst healthcare professionals, comes largely from the prime route of transmission - intravenous drug use. Patients often fear either a hostile reaction from their family doctor or may not want it recorded in their medical records for fear of a prejudicial reaction from those having access to their records and in obtaining insurance. Patients may prefer the more anonymous GUM clinic as a consequence.

In order to facilitate more people being tested and offered treatment, it is important for patients to feel safe in disclosing what for them might be a situation that could provoke anxiety and uncertainty. They often require reassurance regarding a whole host of issues before agreeing to be tested. They may need counselling about the medical and social implications of a hepatitis $\mathrm{C}$ diagnosis, and in particular they may need information about how such a diagnosis may affect work, family and sexual relationships (see above). More needs to be done by the medical profession as a whole with respect to these needs. However, there are signs of improvement, as there is increasing medical awareness of hepatitis $\mathrm{C}$ via ongoing education programmes.

\section{Clinical audit}

Audit is a useful tool in the management of any condition and is an aid to improve current practice. Given that so many patients with hepatitis $\mathrm{C}$ have yet to be diagnosed, it goes without saying that huge improvements could be made merely by identifying those at risk. Now that general practice records are largely computerised, it is a relatively easy task to produce lists of patients who have risk factors for hepatitis $\mathrm{C}$ recorded in their notes thereby identifying a target list for follow up. Annotating their records with an alert could highlight very simply that the

More needs to be done by the medical profession as a whole with respect to these needs. However, there are signs of improvement, as there is increasing medical awareness of hepatitis $C$ via ongoing education programmes.
Huge improvements could be made merely by identifying those at risk. 
New patterns of working may need to be established to deliver treatment in the most effective fashion. subject needs to be broached at the next available opportunity. As the average patient consults about three or four times a year, this sort of semi-opportunistic screening should yield significant results within a relatively short time. The problem is that in general practice there are so many competing interests for such audits and currently, it would probably require someone with an interest in hepatitis $\mathrm{C}$ within the practice for this to be undertaken.

\section{Future directions}

A significant number of patients with HIV and/or HBV are coinfected with HCV. The success rate of clearing hepatitis $\mathrm{C}$ from coinfected individuals is significantly reduced compared with individuals who are infected solely with HCV; SVR rates for hepatitis C in coinfected individuals range from 15 to $29 \%$ for genotype 1 and from 42 to $62 \%$ for genotypes 2 and 3. Morbidity from hepatitis $\mathrm{C}$ is rapidly accelerated by the presence of HIV infection such that there may be more of a threat to a coinfected patient from hepatitis $\mathrm{C}$ than HIV if the HIV infection is stabilised on medication. Therefore, if clearance of HCV can be achieved, it can dramatically reduce the morbidity from hepatitis $\mathrm{C}$, and is well worth attempting, provided that HIV is under control. Increasing numbers of these patients are being jointly managed between the GUM clinic and hepatology.

In some centres in the UK there is a move toward treating some active intravenous drug users, a scenario which used to be a barrier to treatment. Providing these individuals are in a stable lifestyle and are not putting themselves at risk of reinfection, then a small number can been offered treatment based in their drug unit. Such a policy is still at an early stage and numbers being treated in this way remain small. However, if this approach is successful it may well be a useful way of rapidly increasing the numbers taking up treatment. Indeed, this policy is in line with the recent updated NICE guidance on the management of mild chronic hepatitis C.

The better educated the medical profession and general public are, then the numbers needing screening and treatment will undoubtedly increase. There is potentially a huge number of individuals who need treatment and this may swamp existing capacity. Therefore, new patterns of working will need to be established to deliver treatment in the most effective fashion. For example, rather than patients making long trips to hospital, it may be more practical for nurse specialists to be based in the community and be supervised by a GP with a special interest in hepatitis C. In turn, the GP would be overseen by a regional consultant who could provide advice on the more difficult cases and complications. As the treatment model for hepatitis $\mathrm{C}$ is so well proven and protocol driven, in practice such an approach should not prove too difficult to implement. Indeed, if this plan were put in place it is likely that there would be increased uptake merely as a result of its implementation. The patient group most affected are not the sort to sit comfortable in hospital surroundings and might find a community setting more appealing to them.

Lots of research is also ongoing into the development of new drugs for treating hepatitis $\mathrm{C}$ which will undoubtedly result in changes to the currently used protocols. Whatever the overall perspective, the future looks busy! 


\section{Key points}

- Up to half a million people are living with hepatitis $C$ in England and Wales. However, in 2005 , less than $10 \%$ of patients received a diagnosis and an even smaller proportion ( $1.5 \%)$ received treatment.

- There is a need for greater awareness of hepatitis $C$ amongst both the medical community and the general population in order to increase the proportion of patients who are diagnosed and treated and to reduce the likelihood of a future epidemic of liver disease.

- A major challenge in primary care is to identify as many cases of hepatitis $C$ infection as possible by adopting a high index of suspicion and targeting at-risk groups. However, it can be challenging to achieve an accurate diagnosis as acute infection is usually subclinical whilst symptoms of chronic hepatitis $C$ infection are often nebulous or non-existent.

- Effective treatment in the form of pegylated interferon and ribavirin is available and can have a cure rate of up to $80 \%$. It is also cost-effective for the management of mild chronic hepatitis $C$ infection according to recent NICE guidance.

- Treatment of hepatitis C may be performed outside of specialist centres in clinics run by appropriately trained specialist nurses with close involvement of specialist consultants.

- Primary care has an important role to play in managing side-effects from treatment and providing appropriate education. Therefore, close liaison between the GP and the hospital team is important whilst the patient is on the treatment programme.

- Clinical audit can improve the management of hepatitis C. For example, lists of individuals with recorded risk factors for hepatitis $C$ can be generated and these patients can be targeted for appropriate follow up at subsequent consultations.

- New patterns of working and greater collaboration between primary and secondary care will allow treatment to be delivered in the most effective fashion. 